\title{
MANAGEMENT OF A RARE CASE OF NEW ONSET REFRACTORY STATUS EPILEPTICUS IN A DISTRICT GENERAL HOSPITAL
}

\author{
Katyayani K, Kapoor R
}

Department of Anaesthetics \& Intensive Care, East Kent Hospitals University NHS Foundation Trust

New onset refractory status epilepticus (NORSE) is status epilepticus which has continued or recurred despite general anaesthesia for 24 hours or more. The infrequency with which it is encountered and limited evidence base available for treatment strategies make it a formidable management challenge.

\section{CASE REPORT}

- 28 year old previously fit lady developed sudden onset refractory generalized seizures

- Medical management failed to control the seizures

- General anaesthesia (GA) with Intubation and Ventilation therefore commenced on Intensive Care

- Despite the GA and multiple antiepileptic drugs the EEG detected continued epileptic activity

- CSF examination and serological tests for various infectious, autoimmune and toxic etiology were negative

- CT scan and MRI Head failed to show any organic cause for the refractory seizures

- Ketamine infusion, hypothermia, trans cranial electromagnetic stimulation, immunoglobulins and steroids and ketogenic diet attempted as seizures remained refractory.

- After 46 days transferred to tertiary center

- MRI, PET scan, brain and muscle biopsy, mitochondrial and POLG gene sequencing, enzyme assay failed to identify cause

- ITU stay complicated by sepsis (Burkholderia Gladioli)

- After 63 days sedation gradually weaned off

- Six months later seizures gradually improved

- Currently has 10-15 brief focal clinical seizures per day

- Slow cognitive improvement and making progress on the rehabilitation ward.

\section{REFERENCES}

1. Simon Shorvon et al: The treatment of super - refractory status epilepticus: a critical review of available therapies and a clinical treatment protocol. Brain2011:134; 2802-2818.

2. Gaspard N et al. New-onset refractory status epilepticus: Etiology, clinical features, and outcome. Neurology. 2015 Nov 3;85(18):160413 .

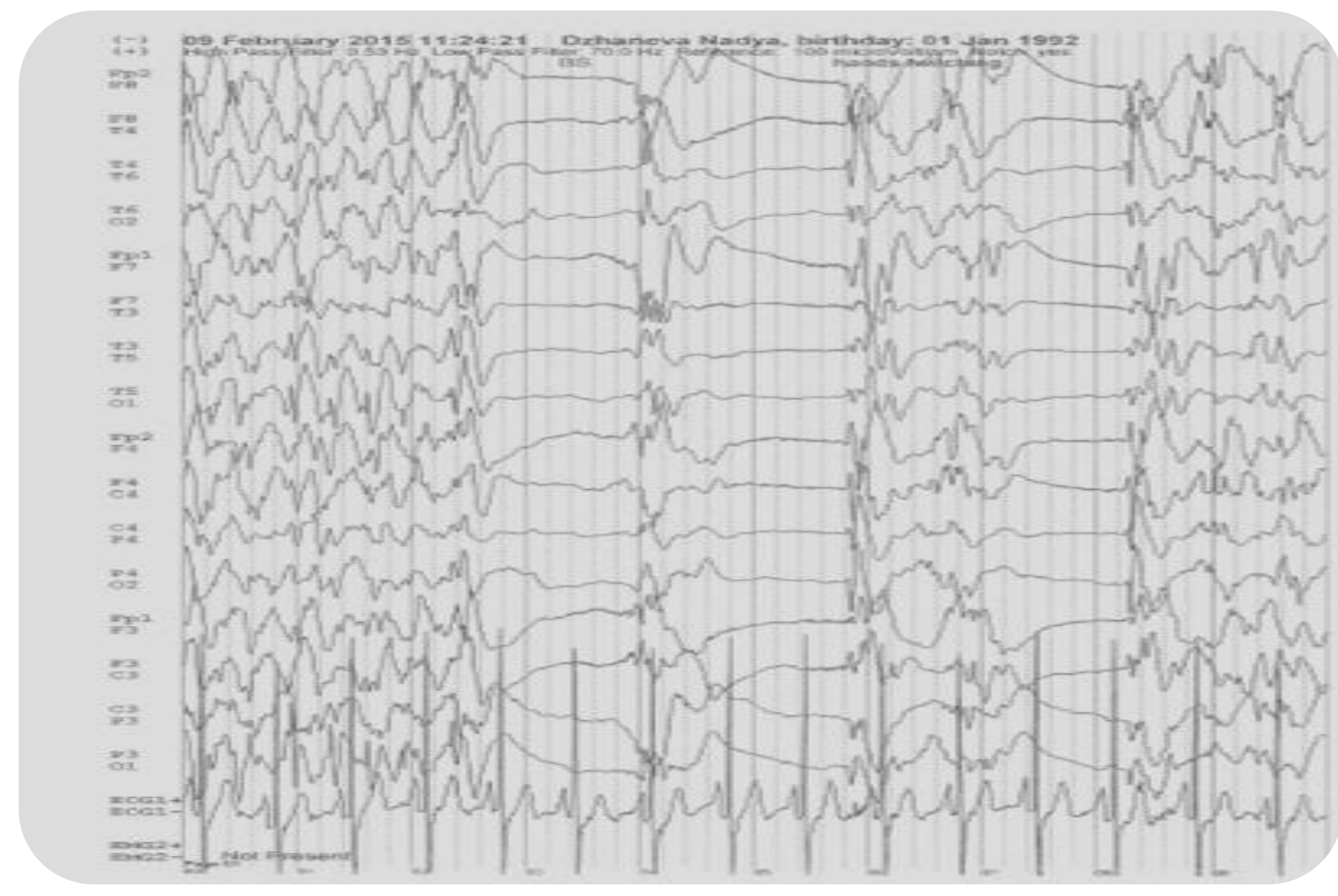

\section{DISCUSSION}

- Approximately $15 \%$ of all cases with status epilepticus admitted to the hospital will become super refractory ${ }^{(1)}$.

- Most commonly identified etiology are autoimmune, para-neoplastic and infective encephalitis, but most often remains cryptogenic ${ }^{(2)}$ (as was in our case)

- Treatment options have been suggested mainly based on clinical experiences (limited evidence base).

- First line: -Polytherapy with anti epileptic drugs

- $\quad$ - Administration of general anaesthetic

- Emphasis should also be on supportive ITU care, identification and treatment of cause

- Second line:

- Hypothermia - Steroid / immunotherapy

- Ketogenic diet - Transcranial magnetic stimulation

- Magnesium - Vagal/deep brain stimulation

- Pyridoxine - Resective neurosurgery

- CSF drainage / ECT

- Outcomes remain poor. With only 35\% patients recovering to baseline and mortality rate of $35 \%{ }^{(1)}$.

- Predictors of outcome include duration of SE, medical complications and use of anaesthetics ${ }^{(2)}$

\section{CONCLUSION}

This case report emphasizes the fact that recovery can occur after weeks or months of Status Epilepticus especially in cases where no cause has been identified. Hence it is of paramount importance that therapy is continued and premature withdrawal of care is not contemplated. 